\title{
Enfoque intercultural a través de una experiencia en física
}

\section{Cross-cultural approach through an experience in physics}

DOI: $10.46932 / \mathrm{sfjdv} 2 \mathrm{n} 2-042$

Received in: january 1st, 2020

Accepted in: March 30th, 2020

\author{
Silvia Inés del Valle Navarro \\ Doctora en Física (FACEN-UNCA)
}

Titular Facultad de Ciencias Exactas y Naturales, Universidad Nacional de Catamarca, Argentina Avda. Belgrano $\mathrm{N}^{\circ} 300, \mathrm{CP} 4700$, Catamarca, Argentina

E-mail: silvina.facen@gmail.com

\author{
Maria Luz del Valle Quiroga \\ Profesora en Física (FACEN-UNCA) \\ Colegio del Carmen y San José \\ Junín N709, CP 4700, Catamarca, Argentina \\ E-mail: tanuquiroga@gmail.com
}

Guillermo Nolasco Leguizamón

Licenciado en Física

Facultad de Ciencias Exactas y Naturales, Universidad Nacional de Catamarca, Argentina

Avda. Belgrano $\mathrm{N}^{\circ} 300$, CP 4700, Catamarca, Argentina

E-mail: guillermo2182002@yahoo.com.ar

Teresita Elisabet Humana

Doctoranda en Energías Renovables

Licenciada en Física

Facultad de Ciencias Exactas y Naturales, Universidad Nacional de Catamarca, Argentina Avda. Belgrano $\mathrm{N}^{\circ} 300, \mathrm{CP} 4700$, Catamarca, Argentina

E-mail: terehumana@gmail.com

Gustavo Adolfo Juarez

Doctorando en Ciencias Humanas

Licenciado en Matemática

Titular Facultad de Ciencias Exactas y Naturales, Universidad Nacional de Catamarca, Argentina Avda. Belgrano $N^{\circ} 300, \mathrm{CP} 4700$, Catamarca, Argentina

E-mail: juarez.catamarca@gmail.com

\section{RESUMEN}

La enseñanza de la Física, como ciencia de la naturaleza, puede ser implementada de manera interdisciplinaria, adaptándose a la formación que ofrece cada carrera según su titulación y al ambiente de ejecución, por ello aquí exponemos la experiencia en biología bajo una mirada intercultural. La búsqueda del conocimiento como objeto de trabajo, aplicado a una investigación de referencia teórica práctica que desarrolla representaciones mentales, campos conceptuales, lenguajes semióticos y situaciones pedagogías, induciendo a estudiar diversos procesos, presentes en la naturaleza y en el orden socio-cultural establecido, se la considera bajo la metodología dada por la etnociencia. El objetivo es desarrollar experiencias en contextos socioculturales diversos a fin de asimilar conceptos nuevos, 
mediante actividades que infieren el estudio de la termodinámica. Siendo el propósito reflexionar cómo estos conocimientos pueden llevarse al aula, integrando la cultura regional valorizando sus conocimientos y legado bajo prácticas etnometodológicas.

Palabras clave: Interculturalidad, Etnometodología, Experiencia, Termodinámica, Estratégia didáctica.

\begin{abstract}
The teaching of Physics, as a science of nature, can be implemented in an interdisciplinary way, adapting to the training offered by each career according to its degree and the environment of execution, so here we expose the experience in biology under an intercultural view. The search for knowledge as an object of work, applied to a theoretical-practical reference research that develops mental representations, conceptual fields, semiotic languages and pedagogical situations, inducing the study of diverse processes, present in nature and in the established socio-cultural order, is considered under the methodology given by ethnoscience. The objective is to develop experiences in diverse socio-cultural contexts in order to assimilate new concepts, through activities that infer the study of thermodynamics. The purpose is to reflect on how this knowledge can be taken to the classroom, integrating the regional culture, valuing its knowledge and legacy under ethnomethodological practices.
\end{abstract}

Key words: Interculturality, Ethnomethodology, Experience, Thermodynamics, Didactic strategy.

\title{
1 INTRODUCCIÓN
}

En la actualidad, el cambio social ha producido un arraigado debate en las diversas prácticas culturales en la que esto lleva a una asimetría y preponderancia en lo natural y económico, como en lo cultural y civilizatorio. Es decir, que dentro de la filosofía intercultural se introduce el aspecto de la culturalidad en la que se somete a una crítica profunda todo tipo de etnocentrismo y universalismo que llevan a poner una base filosófica a la convivencia humana y ecológica, en la que tiende a aproximarse más a los planteamientos posmodernos de la diversidad y pluriculturalidad. Esto lleva a que los estudiantes desarrollen un alto grado de capacidad de observación, sensibilidad y de comunicación que son obtenidos a través de los grandes avances técnico - científicos y que tienen como finalidad aportar a una mejor educación, en la escuela, la universidad y en la sociedad.

La ciencia se ubica entre los demás saberes que las distintas sociedades han originado a lo largo de la historia y los que coexisten actualmente. Dumont (1983) emplea una analogía:

\footnotetext{
Al igual que las regularidades simples de la física clásica aparecen como casos particulares de una perspectiva más amplia, de igual manera lo abstracto universal de la ciencia puede aparecer como un caso particular de lo concreto universal.
}

Esto significa que las diferencias entre una y otra forma de conocer no son cualitativas, sino meramente cuantitativas. En la actualidad, el avance de la ciencia ha propiciado el conocimiento y a las exigencias del sistema económico dedicado a la producción masiva de nuevos productos de consumo y a 
la creación de nuevas necesidades. Los contextos que cada cultura utiliza para explicar los fenómenos naturales tienen el mismo sentido, de donde fueron generadas por lo que no se puede limitar el concepto de una a otra. Latour (1991) expresa:

Ya no hay culturas - diferentes o universales - ni naturaleza universal. Sólo hay naturalezas cultura, y son éstas las que proporcionan la única base de comparación posible.

Se trata de establecer una relación intercultural equitativa y justa, aceptando la equivalencia entre la ciencia en nuestro caso y cada cultura, incluyendo su saber, por lo que es imprescindible entablarlo bajo la forma de un diálogo entre iguales (Carrillo Trueba, 2002).

Por tanto, el trastocamiento que se produce en la formación cognitiva del estudiante al suponer la representación del conocimiento, como objeto de trabajo para ser aplicado a una investigación de referencia teórica - práctica que desarrolla representaciones mentales, campos conceptuales, lenguajes semióticos y situaciones pedagogías, induciendo a estudiar diversos procesos, presentes en la naturaleza y en el orden sociocultural establecido (Grosso, 2008).

Aquí proponemos partir de las disciplinas tradicionales, como por la etnociencias, la cual surgió en 1950, donde el prefijo etno describe al sistema de conocimientos y percepciones propias de una determinada sociedad. Murdock (1965) en su libro titulado Ideas sobre la naturaleza y el hombre, define la palabra etnociencia como:

\footnotetext{
Enunciados generales que comprenden varias clases de nociones especulativas y populares acerca de los fenómenos del mundo exterior y del organismo humano. Luego, subdividió este campo en etnometeorología, etnofísica, etnogeografía, etnobotánica, etnozoología, etnoanatomía, etnofisiología, etnopsicología y etnosociología.
}

En ésta primera definición surge una contradicción que la etnociencia intenta todavía resolver, medio siglo más tarde. Por una parte, el relativismo cultural del autor (postulado básico de la antropología moderna) le hace colocar a la cultura occidental junto a las culturas entonces llamadas primitivas. Sin embargo, Murdock no evita con ello una forma insidiosa de etnocentrismo, pues jerarquiza los saberes de unos y otros: en el rubro llamado ciencias exactas y menciona la lógica, la filosofía, la ciencia pura y aplicada. Además, subdivide estas nociones especulativas y populares de la misma manera que la ciencia occidental, simplemente agregándoles el prefijo etno, a pesar que nada permite suponer, a priori, que otros sistemas de conocimiento tengan la misma estructura interna que el nuestro (Beaucage, 1999).

Garfinkel (2006) considera que el término etno expresa que los miembros de una sociedad tienen disponibles para su uso ciertos conocimientos que son del sentido común de esa sociedad, conocimientos sobre cualquier cosa. Esto nos lleva a considerar que la etnometodología, se refiere a un método que la gente posee. Todos los procedimientos por los cuales las propiedades lógicas y metodológicas de las 
prácticas y los resultados de las investigaciones son tomados en cuenta en sus características generales, son de interés como fenómenos para estudio etnometodológico, pero no de otra manera. Las actividades prácticas organizadas de la vida cotidiana que difieran estructuralmente deben ser buscadas y examinadas en cuanto a su producción, orígenes, reconocimiento y representaciones de prácticas racionales. Toda propiedad de acción lógica y metodológica, cada característica del sentido de una actividad, de su facticidad, objetividad, explicabilidad y de su comunidad debe ser tratada como un logro contingente de prácticas comunes socialmente organizadas. Es recomendable la política de que cualquier escenario social sea visto como auto organizador, con respecto al carácter inteligible de sus propias manifestaciones como representaciones o como evidencias del orden social. Cualquier escenario organiza sus actividades para hacer de sus propiedades un ambiente organizado de actividades prácticas, detectable, contable, informable, narrable, analizable, en resumen, explicable, de las observaciones hechas de pasada, de los relatos admonitorios y similares.

Prudente (2010) expresa a la etnofísica como los saberes populares acerca del conocimiento físico, siendo un campo de estudio que emerge lentamente en el mundo actual. Significa considerar ontológicamente el modo de ver, interpretar, comprender, explicar, compartir, trabajar, lidiar, sentir fenómenos físicos. Es así, un trabajo pedagógico que requiere la apropiación de la memoria cultural del sujeto investigado (Ramalho de Souza, 2013).

Por tanto, dentro de las prácticas etnometodológicas caracterizadas por el análisis de las actividades cotidianas, racionalmente visibles y reportables para todos los efectos prácticos consideramos a la etnofísica que describe la relación entre conceptos empíricos de los fenómenos naturales en un movimiento armónico con la Física. De esta manera, se revalora los significados que poseen los estudiantes que construyen de un modo interrelacional las representaciones de la realidad, y utilizan los instrumentos intelectuales haciendo posible esta construcción. Se destacan los aspectos socioculturales que llevan a una integración en la ejecución de proyectos interdisciplinarios promoviendo la progresiva autonomía de los estudiantes, y en la que el docente orienta la resolución del problema con aspiraciones científicas de las tareas en ejecución, haciendo posible una enseñanza más factible.

\section{PROPUESTA DIDÁCTICA}

En este trabajo se presenta una propuesta didáctica de carácter disímil en el planteo del problema real, que aborda los conceptos implícitos de termodinámica y su vínculo con el aspecto etnofísico relacionado al contexto sociocultural de la región.

Se describe dicha propuesta basada en el desarrollo de una guía de laboratorio que contiene una secuencia de actividades relacionada al estudio de la termodinámica aplicada al proceso crecimiento de 
una planta (Giancoli, 1997; Young y otros, 2009; Curtis y otros, 2008). En la misma, se incluye la noción de etnofísica vinculada a las apreciaciones subjetivas del estudiante en relación, por un lado al carácter didáctico de la tarea y por otro a los aspectos socioculturales de la región donde habita.

El objetivo es desarrollar experiencias en contextos socioculturales diversos, a fin de asimilar conceptos nuevos mediante actividades que infieren en el estudio de la termodinámica.

En consecuencia, la labor del docente no es tratar de trasmitir conocimientos, sino conducir el proceso de aprendizaje, de manera de posibilitar que los estudiantes construyan sus propios significados. Esto no se debe confundir con la llamada enseñanza por redescubrimiento (Moreira y otros., 1993).

Es importante destacar, que la relación con el entorno natural en el que vivimos es uno de los aspectos importantes a tener en cuenta en el desarrollo cognitivo del estudiante, por ello surge la intención de acceder desde la clase de física, a la aplicación de la etnofísica, para abordar los aspectos pedagógicos relacionados a la metodología didáctica en contexto intercultural, que tiene un carácter integrador proveniente del vínculo que se genera entre docente - estudiante y su contexto sociocultural, resultando importante para la investigación que anhela entrelazar las relaciones entre el conocimiento científico adquirido en la universidad y su entorno social.

Esto lleva a considera que la información adquirida y transmitida al estudiante, junto con una actitud constructiva generan acciones que pueden cambiar la visión de la sociedad sobre el entorno que lo rodea, propiciando un cambio de comportamiento significativo en la forma de pensar, actuar y ser responsables con el medio ambiente (Martelli, 2020).

\subsection{DESARROLLO DE LA ACTIVIDAD}

La actividad fue realizada con la modalidad de investigación básica, en la que participaron los alumnos de $2^{\circ}$ Año $\left(2^{\circ}\right.$ cuatrimestre) de 2016 que cursaron la asignatura Física Biológica de las carreras de Biología de la Facultad de Ciencias Exactas y Naturales - UNCA. La población con la que se trabajó fue un total de 50 (cincuenta) alumnos siendo la mayoría de ellos oriundos del interior de la Provincia de Catamarca-Argentina (Figura 1)

Dicha guía de laboratorio estuvo orientada al desarrollo de la unidad didáctica Termodinámica donde se analizó: dimensiones y cinemática del crecimiento de la planta, variación genética, composición física - química, condiciones ambientales y el vínculo sociocultural con la región.

En consecuencia, se procedió a la ejecución de la experiencia en la que se verificaría el crecimiento de la planta (a elección) en el período de dos meses (setiembre y octubre) dentro del cuatrimestre del dictado de la asignatura. Esta elección estuvo regida por la disponibilidad del estudiante, que en algunos casos inicio su experimento utilizando semilla y en otros una planta recién germinada. 
Para iniciar la actividad debía seguir los siguientes pasos: a) Examinar la muestra de suelo que utilizó teniendo en cuenta las características del suelo y la capacidad de retención de agua. b) Escoger una maceta pegando en su parte lateral una regla de $30(\mathrm{~cm})$, y a continuación rellenar la maceta con el suelo seco que analizó, completando la plantación de su semilla, e indicando la altura que corresponda a las raíces y al crecimiento de la misma. Fotografiar el inicio y las posteriores mediciones (Figura 2(a)). c)

Confeccionar una tabla con el registro de datos para llevar el control respectivo de la longitud de crecimiento del tallo, tamaño de las hojas (ancho y largo), temperatura y humedad. d) En dicha tabla se debía agregar la información correspondiente al nombre común de la planta y el nombre científico de la misma y las características de la región donde se llevó a cabo la experiencia. Luego con los datos obtenidos se procede a calcular y graficar la velocidad de crecimiento de la planta (Figura 2 (b)).

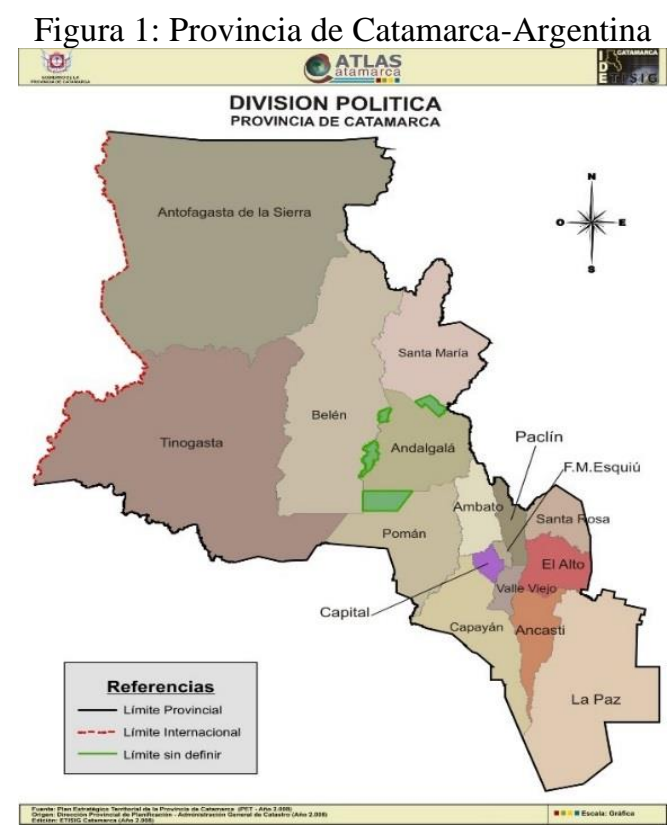

Figura 2: a) Registro fotográfico del crecimiento de la planta, b) Velocidad de crecimiento del tallo
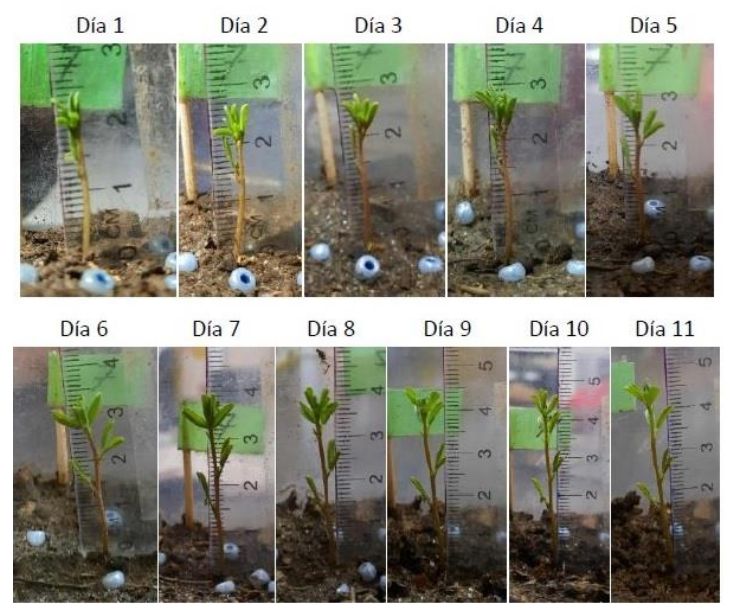

(a)

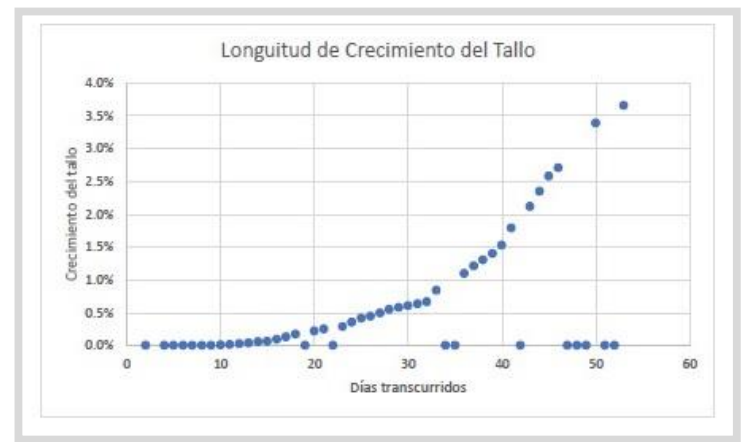

(b) 
Para iniciar ésta tarea, se comenzó realizando la investigación que consistió en la búsqueda bibliográfica para consolidar los conocimientos adquiridos en clase, luego plasmarlo en el proceso de construcción del conocimiento, siendo que esta subjetividad no se negará, mientras sirva como materia prima para el investigador novel. Además, se procedió a explicar a los estudiantes que el objetivo de ésta investigación también se orientaba a reflexionar sobre los aspectos que abarca la Etnofísica, en relación con las distintas ciencias que conlleva a una visión más amplia en la comprensión del tema que se iba a tratar y que está vinculado a nuestro quehacer diario. Dicha experiencia dependía de la participación activa de los estudiantes, pues se requería de ellos una dedicación mayor que la habitual, se les propuso una tarea programada que consistía en realizar una experiencia atípica donde se ponía en evidencia las contradicciones entre sus ideas previas y los fenómenos que estaban a punto de observar. Si bien, los estudiantes declararon su interés en el tema, pues consideraban que era importante para su formación y para poder construir y desarrollar ellos mismos una experiencia de trabajo en un sentido no restrictivo y creativo.

A partir de esto, se puso en marcha el armado de la experiencia en la que se utilizaron elementos auxiliares. Sin embargo, la actividad requería mucho tiempo de observación y dedicación según lo que los mismos estudiantes manifestaron "era complicado disponer de ese tiempo debido a las obligaciones con otras actividades académicas y sociales, pero la tarea era interesante”. Además, algunos estudiantes a su pesar, tuvieron que repetir la experiencia como consecuencia del descuido (la planta se secó y murió). Consecuentemente, se realizó el seguimiento de la experiencia que contaba con la recolección de datos, registro de fotos, manifiesto del transcurrir de su vida cotidiana mientras avanzaba la experiencia y el análisis de los resultados obtenidos.

Para llevar a cabo ésta experiencia se requiere del registro de un alto número de muestras para acercarse a la medida real del crecimiento de la planta. Porque las plantas son organismos modulares. Esto implica que su crecimiento ocurre mediante la repetición de módulos. Cada módulo consiste en una hoja con una yema axilar y el punto en donde se insertan las hojas son llamados nudos. Entre nudo y nudo hay un entrenudo. De esta forma, el crecimiento de las plantas consiste en la repetición de este patrón y en la expresión de las yemas axilares en apéndices reproductivos (flores) o vegetativos (ramas). El crecimiento en longitud y grosor se da gracias a la actividad de los meristemas. Algunos de estos tejidos vegetales se encuentran localizados en la zona apical, meristemas apicales, que se encargan de promover la síntesis de auxinas que optimizan el desarrollo de los órganos de las plantas. Además de auxinas, se encuentran otro tipo de hormonas sintetizadas en las raíces que estimulan la división celular, inhibiendo el envejecimiento de hojas y flores, llamadas citoquininas. Tanto auxinas como citoquininas, regulan el desarrollo y la actividad metabólica de las plantas (Curtis y otros, 2008). 
Por otro lado, el trabajo etnográfico en el aula duró todo el cuatrimestre y al finalizar el mismo, se realizó la evaluación prevista que consistió en la presentación de los resultados de la investigación en una mesa redonda semiestructurada; donde los integrantes de cada grupo manifestaron sus reflexiones y algunas causas que a su entender contribuyeron a esta situación en relación con su contexto sociocultural y su universo de significados. Reconociendo que la etnometodología se refiere a métodos que posee la gente, siendo conocimientos de asuntos cotidianos que pueden ser revelados en forma de razonamiento prácticos, pues en toda investigación del mundo real incluso en eventos físicos o biológicos el quehacer sociológico es una referencia a las actividades organizadas de la vida cotidiana.

La mesa redonda semiestructurada fue orientada en base a las siguientes preguntas:

1. Explique ¿Cuál son los factores que producen cambios en la forma, dimensión y velocidad de la planta a lo largo de su crecimiento?

2. ¿Cuál fue el resultado de las observaciones del experimento?

3. ¿Cuál es la respuesta de la planta a los estímulos que se haya expuesta?

4. ¿Cuáles son las hormonas vegetales que regulan y coordinan las funciones vitales de la planta, y además como verifica que cumplen con las leyes de la termodinámica?

5. ¿De qué manera puede integrar ésta propuesta, a su entorno y contexto sociocultural?

6. ¿Cuál es la expectativa que manifiesta al realizar este tipo de experiencia desde el punto de vista de la etnofísica?

A continuación, se detallan algunas conclusiones obtenidas por los miembros de los grupos participantes en la que se respetan sus propias opiniones en relación a las preguntas realizadas:

GRUPO I: (los integrantes son oriundos de San Fernando del Valle de Catamarca):

Elegimos la semilla de algarrobo, nombre científico: ceratonia siliqua, siendo una planta autóctona de Catamarca, es un árbol de hasta $10 \mathrm{~m}$ de altura, aunque su altura media es de 5 a $6 \mathrm{~m}$, y de follaje perenne. Esta depende de algunos factores que influyen en su crecimiento como la temperatura, luz solar, presión atmosférica, agua y nutrientes, y otros que la desfavorecen como el exceso de luz que hace que las hojas se chamusquen o presenten manchas pardas, las hojas también pueden disminuir de tamaño y perder el color verde por uno más blanquecino o amarillo claro, y algunas por demasiado calor se queman y se secan. Por falta de luz, algunas se tornan amarilla y empiezan a caerse. Además tiene la característica de presentar hojas bipinnadas de color verde oscuro con una dimensión de entre 10 y 20 cm de largo y sus flores son pequeñas, rojas y sin pétalos. El fruto, llamado algarroba o garrofa, es una vaina coriácea de color castaño oscuro de 1 a $3 \mathrm{dm}$ de longitud, que contiene una pulpa gomosa de sabor dulce y agradable 
que rodea las semillas. Hicimos el seguimiento de su crecimiento, en la que se destaca que a partir del $7^{\text {mo }}$ día se ve que la semilla salió de la tierra (una parte de ella se ve un brote que es la radícula que está sumergida en la tierra y la otra parte está envolviendo las primeras hojas que son los cotiledones). Luego, se continua registrando los datos del crecimiento, a los 18 días se observa en el medio de los cotiledones un pequeño brote que son verdaderas hojas de la planta que empiezan a crecer; a los 20 días las verdaderas hojas se abren por completo; a los 22 días las hojas verdaderas tienden a cerrarse de noche y abrirse de día, cuando está la luz del día; a los 25 días el crecimiento de la hoja fue mínimo de 0,2 cm, y a los 27 días la longitud alcanzada por el tallo es de 7,5 cm y en el medio del tallo de las hojas verdaderas se observan pequeños brotes. De aquí se concluye que la velocidad de crecimiento es paulatino respectando la formación de los pequeños brotes.

Nuestra elección se debió a que uno de nuestros compañeros, tiene en su casa un vivero -que junto a su padre que es agrónomo arman los mudrones para la venta- esto nos dio la posibilidad de compartir los conocimientos que nos aportó el padre de nuestro compañero, para lograr tener una planta muy saludable con el cuidado adecuado; ya que es muy usada como recurso alimenticio (la harina de algarroba se la utiliza para obtener diversos productos como el patay, turrones de miel, licor, etc.) y además las vainas de éste árbol son usadas para forraje. En relación a la Etnofísica nos resultó importante, ya que nos permitió vincularnos entre nosotros y aprendimos de qué manera la física interviene en los distintos procesos biológicos que infieren en todo ser vivo, y de cómo la naturaleza se las arregla para conseguir el equilibrio natural a pesar de tantos contaminantes existentes en nuestra atmosfera.

GRUPO II: (los integrantes son oriundos del Departamento Andalgalá):

Utilizamos la semilla vincapervinca de Madagascar, nombre científico: vinca rosea. Es una planta originalmente perenne, pero que ha sido cultivada como anual; la misma se adaptó al clima que posee el Departamento Andalgalá, no solo por la temperatura sino también por la salinidad del agua de la región. El crecimiento de esta planta depende de varios factores (energía lumínica, temperatura, fertilizantes, en este caso se utilizó compost fabricado por nosotros). Así fuimos observando con el correr del tiempo, que la planta va creciendo a medida que va absorbiendo nutrientes del suelo, la energía lumínica y el cuidado; también se presentó el cambio de clima en algunos días, ya que hubo altas y bajas temperaturas, pero se mantuvo constante el crecimiento en longitud, en los días con más calor nos pareció que hubo un crecimiento un poco más rápido. Por lo tanto, la velocidad variable que depende de la temperatura que es un factor importante para el crecimiento, el riego y la iluminación, pudiéndose completar el proceso de fotosíntesis para la fabricación de nutrientes. 
En nuestra opinión, consideramos que esta experiencia fue bastante interesante, ya que aprendimos a realizar una investigación y a tener en cuenta los consejos que nos aportaron algunos de nuestros parientes (abuelos y tíos) en el cuidado de ésta planta, que no es originaria de la región norteña pero que se adapta bastante bien. El tema de Etnofísica primera vez que lo escuchamos y nos pareció que no es tan difícil entender el aporte que puede hacer la ciencia para mejor nuestro ambiente.

\section{GRUPO III: (los integrantes son oriundos del Departamento Belén):}

Elegimos la semilla de maíz, cuyo nombre científico es zea mays, es de porte robusto de fácil desarrollo y de producción anual. Los factores como la temperatura, humedad y los gases (oxigeno o dióxido de carbono) son los que afectan a la germinación de la planta, por ejemplo: si nosotros le modificamos el factor de temperatura el crecimiento de la planta será anormal y puede llegar a morir si no se adapta a la temperatura sometida. El agua cumple una función crucial en la vida de las plantas, ya que en los procesos de fotosíntesis hace que la planta obtengan el dióxido de carbono de la atmosfera, pero al mismo tiempo se exponen a una pérdida de agua y por tanto a una amenaza de deshidratación; para prevenirla, la planta debe absorber agua por las raíces y transportarla a la parte superior, si se produce un pequeño desequilibrio entre la absorción de agua y la pérdida de agua a la atmósfera puede causar déficit hídrico que puede llevar a una mal funcionamiento de muchos procesos celulares de la planta. En cuanto a la velocidad de crecimiento es muy variable ya que depende de varios factores, que acabamos de expresar.

Respecto a esta experiencia llegamos a comprobar haciéndole un análisis químico, donde el maíz posee calcio, fósforo, magnesio, hierro, zinc (que es esencial para la actividad de más de 70 enzimas y forma parte de proteínas que actúan como receptores hormonales e intervienen en el crecimiento) y mayor cantidad de potasio que de sodio. En cuanto a la Etnofísica, fue interesante ya que investigamos que los diaguitas utilizaban la mazorca como alimento fundamental de su dieta por considerarla que presenta un alto valor nutritivo e importantes propiedades medicinales, también usaban el maíz para armar elementos decorativos que servían para ofrendar a los dioses. En la actualidad se usa en la cocina de mil maneras diferentes: cocido, asado, guisado, macerado, tostado, en harina, en tortillas, en forma de granos, en aceite de maíz, etc.; además la fina capa que recubre las aspirinas y otros analgésicos está hecha de almidón de maíz.

\section{GRUPO IV: (los integrantes son oriundos de San Fernando del Valle y del Departamento Pomán):}

La semilla utilizada era de tamaño mediano, textura rugosa, color marrón su nombre común es capuchina enana o taco de reina y su nombre científico es tropaeolum majus. El experimento nos permitió 
observar que existe una íntima relación entre el desarrollo de la planta y las condiciones del ambiente que las rodea. Estas condiciones se refieren a la temperatura, la humedad del aire y del suelo, el tipo de sustrato, la cantidad de luz recibida. Durante el proceso de germinación la semilla recibió humedad a través del papel secante logrando germinar, pero aquellas que quedaron en contacto en el agua en el fondo del germinador, se pudrieron debido al exceso de agua con la consecuente aparición de hongos. Las plantas se desarrollaron en el germinador hasta los 17 días, luego fueron trasplantados a la tierra en donde recibieron los nutrientes necesarios creciendo satisfactoriamente lo que demuestra que ella intercambia sustancias con el suelo a través de las raíces, cuya velocidad fue aumentando progresivamente llegando alcanzar una altura de $7 \mathrm{~cm}$; mientras que las plantas que quedaron en el germinador crecieron débiles y se secaron al poco tiempo. En el germinador se formaban micro gotas evidenciadose la traspiración cuando la temperatura era elevada, algunas hojas se marchitaron y se secaron por ese motivo se ubicó al germinador en un sector con luz indirecta, si bien la planta necesita luz, agua, aire, suelo, pero en la medida justa las temperaturas extremas son nocivas para ella.

En relación a la Etnofísica fue interesante y más si pudimos llevar a cabo éste tipo de experiencia, porque nos mostró que hay otras formas distintas de realizar una investigación haciéndola desde un punto de vista de nuestro quehacer cotidiano, en la que nos enseñó a ser responsables de un ser vivo. Cabe aclarar, que la experiencia la hicimos simultáneamente en San Fernando del Valle y en el Departamento Pomán, lo que nos posibilitó hacer contacto con los agricultores de la zona que nos aportaron sus conocimientos en el manejo de la planta haciendo uso de abonos orgánicos elaborados de manera artesanal. Esto nos permitió comparar resultados y mejorar el cuidado de la planta, sin perder de vista la propuesta planteada.

\section{GRUPO V: (los integrantes son oriundos de Los Ángeles -Departamento Capayán)}

El ejemplar elegido fue la planta de romero, cuyo nombre científico es Rosmarinus o Picinalis sp. En primera instancia, se escogió una planta joven de romero por sus características de crecimiento. Luego se procedió a medir la longitud del tallo y la longitud de la hoja de la planta. Estas medidas fueron diarias durante treinta días. Se le agregaba agua a la planta día por medio y fue colocada en un lugar que recibía luz solar directa necesaria para su crecimiento. Posteriormente, a los veinte días se trasladó la planta a una maceta de mayor tamaño que contenía mantillo para abonarla, permitiendo también el desarrollo normal de sus raíces. A medida que la planta crecía, ésta fue variando en sus dimensiones dependiendo de las condiciones climáticas que afectaron de manera directa a dicho crecimiento. Esto se debe que en días nublados o con ráfagas de viento la planta se mantuvo en un estado de latencia, en él no se produjo aumento de su biomasa y, en aquellos días de sol moderado se obtuvo un crecimiento gradual y normal. 
Cabe destacar que la presencia de luz solar a gran escala afecta en la nutrición de la planta ya que la misma sufre un determinado estrés hídrico lo cual provoca un grado de desorden en la estabilidad del crecimiento.

En relación a la Etnofísica pudimos aprender una forma más interesante para realizar una investigación porque no es solo la aplicación de los conceptos físicos-biológicos aprendidos en la materia, sino también saber cómo realmente las plantas son capaces de captar cambios en el medio donde se desarrolla (estímulos) y reacciona ante ellos, si bien la planta no puede desplazarse, pero responde a los cambios ambientales mediante las nastias y los tropismos.

\section{RESULTADOS}

Como síntesis de los resultados obtenidos de la experiencia del crecimiento de la planta, se determinaron tres factores que participaron en el mismo, siendo estos: los datos registrados (dimensiones de la planta, condiciones ambientales), la aplicación de los conceptos de la termodinámica y los conocimientos aportados por miembros de la comunidad en relación al entorno sociocultural justificados bajo métodos enmarcados en la etnometodología, llevando a los estudiantes a opinar respeto a tales factores que influyen en este experimento, tal como se muestra en la Figura 3.

Dentro de la discusión, se tomaron los aspectos biológicos en la que se considera que el crecimiento de los diferentes órganos de las plantas, es un proceso fisiológico complejo, que depende directamente de la fotosíntesis, la respiración, la división celular, la elongación, la diferenciación de tejidos, entre otros, y que además está influenciada por factores como temperatura, intensidad de luz, disponibilidad de agua y de nutrientes. Durante su crecimiento, la planta presenta los siguientes tropismos en respuesta a los estímulos: fototropismo corresponde a la respuesta de la planta frente al estímulo luminoso (luz solar); hidrotropismo las raíces que buscaban el agua; tigmotropismo respuesta al estímulo proveniente del tacto; y gravitropismo respuesta a estímulos producido por efecto de la fuerza de gravedad.

Desde la física se considera una relación entre las leyes de la termodinámica y la velocidad de crecimiento de la planta que es variable, pues es dependiente de la primera ley "la energía no se crea ni se destruye, sólo se transforma", siendo que ésta se cumple durante la fotosíntesis, en donde las plantas aprovechan la luz del sol (energía lumínica) y la transforma en energía química que será luego utilizada en todos aquellos procesos internos que las plantas lleven a cabo durante el crecimiento y desarrollo. Y en la segunda ley "la energía tiende a pasar de formas más organizadas y concentradas a otras menos organizadas y más dispersas”, lo cual implica que la transferencia de energía no es eficaz y gran parte de la energía se torna tan dispersa que deja de ser útil, es por ello, que la planta toma una pequeña parte de la energía que proviene del sol mientras que la energía restante se pierde y es desaprovechada. 
Es decir, cuando las células comienzan a crecer y reproducirse durante la germinación, parte de las células formadas crece y se diferencia de acuerdo con la función que tendrán en la planta adulta, pero pequeños conglomerados de células se conservarán indiferenciados y retendrán su potencialidad multiplicativa. Estos conglomerados de células se encuentran en diversas partes de la planta en crecimiento: en las yemas de la punta del tallo, de las axilas de las hojas y de las ramas, a veces también en los bordes de las hojas y en la base del tallo, en la punta y en las axilas de las raíces.

En relación a la etnofísica de acuerdo a lo que expresaron los distintos grupos de trabajo se observó una constante reminiscencia a las contribuciones aportadas por los familiares de los estudiantes, donde les enseñaron y mostraron como respetar a la madre tierra y evitar contaminarla con los implementos químicos actuales.

Figura 3: Resultados obtenidos de la experiencia del crecimiento de la planta.

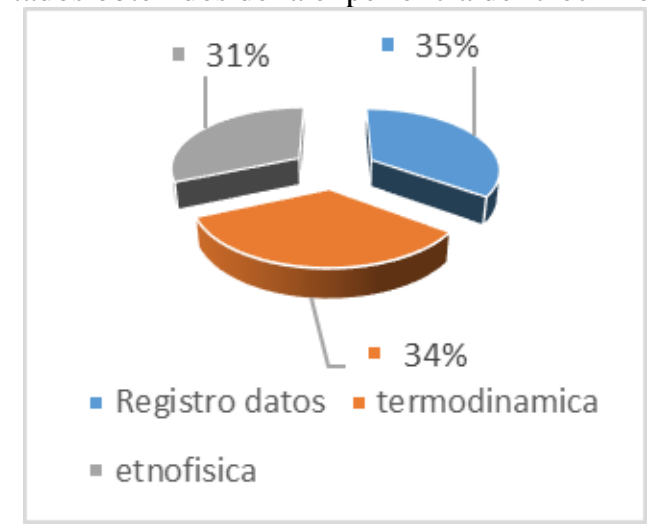

Los resultados obtenidos, muestran que la estrategia didáctica implementada es efectiva para lograr la construcción de conceptos fundamentales en el estudio de la termodinámica. Además, de forma objetiva, se evidencio un sentimiento manifiesto por parte de los estudiantes, de que es posible establecer una relación de la física en vinculación a lo sociocultural, que desde el punto de vista de la etnofísica ésta representa un complejo entramado de significados, apropiaciones, sentidos y compromiso con la sociedad.

\section{CONCLUSION}

Esta investigación muestra la importancia de la tarea didáctica desde la perspectiva etnofísica, abriéndose un abanico de posibilidades temáticas que promueven las comparaciones de los nuevos conocimientos adquiridos por los estudiantes, y que inducen al análisis de las relaciones socioculturales en la que se trata de entender el pensamiento físico que debe ser transpuesto.

El trabajo de campo que se llevó a cabo, estimuló a los estudiantes en el interés por rediseñar nuevas experiencias y de continuar profundizando algunos temas surgidos en esta tarea realizada, para lograr afianzar lo aprendido en una investigación que procura usar referencias teóricas en interpretación 
de resultados, lo cual es importante para desarrollar representaciones mentales, campos conceptuales, lenguajes semióticos y situaciones didácticas en etnofísica. Lahera Claramonte (2009) expresa "la física debe presentar una fisonomía específica, prevaleciendo no lo que debe saber el alumno, sino aquello que no debe ignorar". 


\section{REFERENCIAS}

- $\quad$ Beaucage P. (1999) La Etnociencia, su desarrollo y sus problemas actuales. Universidat de Valencia: Cronos, 3 (1) $47-92$.

- $\quad$ Carrillo Trueba C. (2002) Etnociencias. Ciencia 66 Abril, p. 106 - 117. Universidad Nacional Autónoma de México.

- $\quad$ Curtis H., Schnek B., Massarini A. (2008) Curtis Biología. Editorial Médica Panamericana. (7ma Edición).

- $\quad$ Dumont L. (1983) Essais sur I'individualisme. Paría. Seuil.

- Garfinkel H. (2006) Estudios en Etnometodología. México: Editorial Anthropos. UNAM

- $\quad$ Giancoli, D. (1997) Física. Principios con aplicaciones. México: Prentice Hall Hispanoamericana S.A. (4ta edición)

- Grosso J.L. (2008) Luchas interculturales y políticas del conocimiento. La infrahistoria poscolonial de la educación. En F. Pérez Bonfante. Cátedra Abierta Estanislao Zuleta. Pensar colectivamente la universidad. Santiago de Cali: Universidad del Valle.

- $\quad$ Latour, B. (1991) Nous návons jamais été modernes. Essai d'anthropologie symétrique. Paris, La Découverte.

- $\quad$ Lahera Claramonte J. (2009) Fundamentos de Física para el grado de Magisterio. Una antología de textos y problemas comentados y evaluados. Madrid: Editorial CCS.

- $\quad$ Martelli, A. (2020) Environmental education action with urban tree planting in order to neutralize greenhouse gases. South Florida Journal of Development, Miami, V.1, n.1, 2-10. ISSN 2675-5459

- $\quad$ Moreira M., Calvo Redondo A. (1993) Constructivismo: significados, concepciones erróneas y una propuesta. Memorias REF VIII, p. 237-248.

- $\quad$ Murdock, G. (1965) Outline of Cultural Materials. New Haven, Human Relations Area Files.

- $\quad$ Ramalho de Souza, E.S. (2013) Etnofísica, modelagem matemática, geometria... tudo no mesmo Manzuá. Armazónia. Revista de Educacao em Ciéncias e Matemáticas. V. 9 (18) p.99-112. ISSN (versión online) 2317-5125.

- $\quad$ Young H.D., Freedman R.A. (2009) Sears-Zemansky: Física Universitaria. Vol.1. México: Pearson Educación, (Decimosegunda edición) 